\title{
Comparative analysis of antifibrinolytic medications in pediatric heart surgery
}

\author{
Sara K. Pasquali, MD, MHS, ${ }^{\mathrm{a}, \mathrm{e}}$ Jennifer S. Li, MD, MHS, ${ }^{\mathrm{a}, \mathrm{e}}$ Xia He, MS, ${ }^{\mathrm{e}}$ Marshall L. Jacobs, MD, ${ }^{\mathrm{f}}$ \\ Sean M. O’Brien, PhD, ${ }^{\text {be }}$ Matthew Hall, PhD, ${ }^{\mathrm{g}}$ Robert D. B. Jaquiss, MD, ${ }^{\mathrm{d}}$ Karl F. Welke, MD, MS, \\ Eric D. Peterson, MD, MPH, ${ }^{\mathrm{c}, \mathrm{e}}$ Samir S. Shah, MD, MSCE, ${ }^{\mathrm{i}}$ and Jeffrey P. Jacobs, MD
}

Objectives: Recent studies suggest adverse events associated with aprotinin in adults may not occur in children,
and there is interest in further pediatric study of aprotinin. However, there are limited contemporary data comparing
aprotinin with other available antifibrinolytics (aminocaproic acid [ACA] and tranexamic acid [TXA]) to guide
current practice and aid in potential trial design. We performed a comparative analysis in a large multicenter cohort.

\begin{abstract}
Methods: The Society of Thoracic Surgeons Congenital Heart Surgery Database (2004-2008) was linked to medication data from the Pediatric Health Information Systems Database. Efficacy and safety outcomes were evaluated in multivariable analysis adjusting for patient and center factors overall and in neonates and those undergoing redo sternotomy.
\end{abstract}

Results: A total of 22,258 patients ( 25 centers) were included: median age, 7.6 months (interquartile range, 2.6-43.4 months). Aprotinin (vs no drug) was associated with a significant reduction in combined hospital mortality/bleeding requiring surgical intervention overall (odds ratio [OR], 0.81; 95\% confidence intervals [CI], $0.68-0.91)$ and in the redo sternotomy subgroup (OR, 0.57; 95\% CI, 0.40-0.80). There was no benefit in neonates and no difference in renal failure requiring dialysis in any group. In comparative analysis, there was no difference in outcome in aprotinin versus ACA recipients. TXA (vs aprotinin) was associated with significantly reduced mortality/bleeding requiring surgical intervention overall $(\mathrm{OR}, 0.47 ; 95 \% \mathrm{CI}, 0.30-0.74)$ and in neonates (OR, 0.30; 95\% CI, 0.15-0.58).

Conclusions: These observational data suggest aprotinin is associated with reduced bleeding and mortality in children undergoing heart surgery with no increase in dialysis. Comparative analyses suggest similar efficacy of ACA and improved outcomes associated with TXA. (J Thorac Cardiovasc Surg 2012;143:550-7)

Aprotinin was the most commonly used antifibrinolytic medication in children undergoing heart surgery until it was taken off the market in 2007 after adult studies

From the Departments of Pediatrics, ${ }^{\mathrm{a}}$ Biostatistics, ${ }^{\mathrm{b}}$ Medicine, ${ }^{\mathrm{c}}$ and Surgery, ${ }^{\mathrm{d}}$ Duke University School of Medicine, and the Duke Clinical Research Institute, ${ }^{\mathrm{e}}$ Durham, NC; Department of Pediatric and Congenital Heart Surgery ${ }^{\mathrm{f}}$ Cleveland Clinic, Cleveland, Ohio; Child Health Corporation of America, ${ }^{\mathrm{g}}$ Shawnee Mission, Kan; Mary Bridge Children's Hospital, ${ }^{\text {h }}$ Multicare Health System, Tacoma, Wash; Department of Pediatrics, ${ }^{\text {i }}$ Children's Hospital of Philadelphia, Philadelphia, Pa; and Division of Thoracic and Cardiovascular Surgery, ${ }^{\mathrm{j}}$ Congenital Heart Institute of Florida, All Children's Hospital and Children's Hospital of Tampa, University of South Florida College of Medicine, St Petersburg and Tampa, Fla.

This study was supported by National Heart, Lung, and Blood Institute Grant 1RC1HL099941-01, under the 2009 American Recovery and Reinvestment Act. Dr Pasquali: Grant support National Heart, Lung, and Blood Institute (1K08HL103631-01), and American Heart Association Mid-Atlantic Affiliate Clinical Research Program. Dr Shah: Grant support National Institute of Allergy and Infectious Diseases (K01 AI73729) and Robert Wood Johnson Foundation Physician Faculty Scholar program. Dr J Jacobs: Chair, Society of Thoracic Surgeons Congenital Heart Surgery Database Task Force, and medical advisor and shareholder, CardioAccess. Dr Peterson: Principal Investigator, Society of Thoracic Surgeons National Databases Analytic Center.

Disclosures: Authors have nothing to disclose with regard to commercial support.

Received for publication Jan 18, 2011; revisions received June 3, 2011; accepted for publication June 27, 2011; available ahead of print Jan 20, 2012.

Address for reprints: Sara K. Pasquali, MD, MHS, Assistant Professor of Pediatrics, Duke University Medical Center, Duke Clinical Research Institute, PO Box 17969, Durham, NC 27715 (E-mail: sara.pasquali@ duke.edu).

$0022-5223 / \$ 36.00$

Copyright (c) 2012 by The American Association for Thoracic Surgery doi:10.1016/j.jtcvs.2011.06.048 reporting increased mortality and renal failure. ${ }^{1,2}$ As a result, aprotinin is also no longer available to children undergoing heart surgery. ${ }^{3}$ Recent studies have suggested that the adverse events associated with aprotinin in adults may not occur in children. $\mathrm{We}^{4}$ previously evaluated safety outcomes in more than 30,000 children undergoing heart surgery and found that aprotinin was not associated with increased mortality or dialysis. This previous study was limited by the use of administrative data, focused on safety outcomes only, and did not compare aprotinin with other antifibrinolytic medications (aminocaproic acid [ACA] and tranexamic acid [TXA]). The few previous comparative analyses evaluating these medications have been limited by small sample size. ${ }^{5,6}$

Thus, although there is interest in further prospective study of aprotinin in children, there are limited contemporary data comparing aprotinin with other available antifibrinolytic medications to guide current practice and aid in planning future prospective study. The purpose of the present study was to evaluate the comparative outcomes associated with aprotinin, ACA, and TXA in a large multicenter cohort. We first evaluated outcomes associated with aprotinin versus no drug before 2007 when aprotinin was most widely used. We then subsequently assessed comparative outcomes associated with aprotinin, ACA, and TXA during 


\section{Abbreviations and Acronyms \\ ACA = aminocaproic acid \\ $\mathrm{CI}=$ confidence interval \\ EACTS $=$ European Association for \\ Cardiothoracic Surgery \\ ICD-9 = International Classification of \\ Diseases, Ninth Revision \\ $\mathrm{OR}=$ odds ratio \\ PHIS = Pediatric Health Information Systems \\ STS- $\quad=$ The Society of Thoracic Surgeons \\ CHS Congenital Heart Surgery (Database) \\ TXA $=$ tranexamic acid}

2007 to 2008 when use transitioned from aprotinin to the other 2 medications. We evaluated the overall cohort of children undergoing heart surgery, and the subgroups undergoing redo sternotomy and neonates as these groups may be more prone to bleeding. ${ }^{3,7}$

\section{METHODS}

\section{Data Source}

De-identified data from the Pediatric Health Information Systems (PHIS) Database and The Society of Thoracic Surgeons Congenital Heart Surgery (STS-CHS) Database were used for this analysis. Data from 30 centers participating in both databases from 2004 to 2008 were linked using the method of "indirect identifiers" as previously described and verified. ${ }^{8}$ Overall, data on $90 \%$ of 45,830 eligible patients at these centers were successfully linked using this methodology. Through linking these data, we are able to capitalize on the strengths of both data sets and use the detailed diagnosis and procedure information in the STS-CHS Database and medication data from the PHIS Database, as described below.

The PHIS Database is a large administrative database containing inpatient data from 41 pediatric hospitals in the United States affiliated with the Child Health Corporation of America. The database currently contains information from more than 4.6 million inpatient discharges. Data quality and reliability are assured through a joint effort between Child Health Corporation of America and participating hospitals. Data collected include demographics, diagnoses, and procedures (using International Classification of Diseases, Ninth Revision [ICD-9] coding), in-patient outcomes data, and resource use data from the hospital bill including pharmaceuticals, imaging, laboratory studies, and hospital charges.

The STS-CHS Database is the largest clinical pediatric heart surgery data registry in the world. It currently contains data on more than 160,000 operations performed since 1998. The STS-CHS Database contains preoperative, operative, and outcomes data on all patients undergoing pediatric heart surgery at participating centers. Diagnoses and procedures are coded using the International Pediatric and Congenital Cardiac Code, which was developed through an international collaborative effort of pediatric cardiologists and congenital heart surgeons. ${ }^{9}$ Data quality and reliability are assured through intrinsic verification of data as well as a formal process of site visits and data audits. ${ }^{10}$

This study was approved by the institutional review boards at Duke University Medical Center and The Children's Hospital of Philadelphia with waiver of informed consent. The study was also reviewed and approved by the STS-CHS Database Task Force and Child Health Corporation of America in compliance with their PHIS Database External Use Guidelines.

\section{Study Population}

Thirty centers ( $\mathrm{n}=41,371$ patients with successfully linked data as described earlier) were eligible for inclusion. Centers with more than $15 \%$ missing data for any STS-CHS study variable were excluded $(n=5$ centers); whereas the STS-CHS Database contains nearly complete data for the required standard data fields regarding procedure and in-hospital mortality, not all centers submit complete data for the other variables in the STS-CHS Database, and it is therefore standard practice to exclude centers with more than $15 \%$ missing data for key study variables to maximize data integrity and minimize missing data). This left 25 centers ( $n=32,660$ patients) eligible for inclusion. Patients with missing PHIS medication data $(\mathrm{n}=1805)$ were excluded. STS-CHS data were used to identify patients undergoing any surgery classified in The Society of Thoracic SurgeonsEuropean Association for Cardiothoracic Surgery (STS-EACTS) risk stratification system (category $1=$ lowest mortality risk, category $5=$ highest mortality risk). ${ }^{11}$ This system was recently developed on the basis of empiric data from nearly 80,000 patients. It includes a greater number of operations compared with other risk stratification systems. ${ }^{11}$ Ninety-seven percent of the remaining 30,855 eligible patients were classified in the STS-EACTS system; only those patients undergoing surgery involving cardiopulmonary bypass $(n=22,485)$ were included. Only the first cardiovascular operation of the admission was analyzed, and patients who received more than 1 of the drugs of interest on the day of the operation and those with missing weight data were excluded. This left a cohort of 22,258 patients from 25 centers.

\section{Data Collection}

Data were collected from both the STS-CHS and PHIS databases (as noted below for each variable). These included age (PHIS), prematurity (PHIS, collected for neonates only using ICD-9 codes for gestational age $<37$ weeks), sex (PHIS), race (PHIS), weight (STS-CHS), the presence of any noncardiac/genetic abnormality (STS-CHS), any preoperative risk factor (as defined in the STS-CHS Database), any previous cardiothoracic surgery (STS-CHS), STS-EACTS risk category for the primary procedure performed (STS-CHS), the use of perioperative corticosteroids (PHIS), and center average annual surgical volume of STS-EACTS classified operations (STS-CHS). ${ }^{12}$

\section{Primary Exposure}

The primary exposure variable was receipt of aprotinin, ACA, TXA, or none of these medications on the day of the operation (PHIS).

\section{Outcomes}

The primary efficacy end point was a composite of in-hospital mortality (PHIS) or bleeding requiring surgical intervention during the hospitalization (STS-CHS). These outcomes were also examined individually. Other outcomes evaluated included postoperative total and intensive care unit lengths of stay (combination of STS-CHS and PHIS data). Postoperative duration of mechanical ventilation in days was also collected (PHIS) The primary safety end point was acute renal failure requiring temporary or permanent dialysis (STS-CHS). Owing to reports in adults of possible neurologic side effects of TXA, postoperative neurologic impairment (transient or permanent neurologic deficit or new-onset seizure; STS-CHS) was also evaluated. ${ }^{13}$

\section{Analysis}

Variables were described using standard summary statistics. Unadjusted outcomes were compared between groups using $\chi^{2}$ and Wilcoxon rank sum tests. Multivariable analysis was also performed to evaluate outcomes associated with aprotinin, ACA, and TXA (as described below). All models were adjusted for age, weight z-score, race, sex, any noncardiac/genetic abnormality or other STS-CHS preoperative risk factors, any previous cardiothoracic operation, STS-EACTS surgical risk category, and use of 
TABLE 1. Patient and center characteristics in the overall cohort

\begin{tabular}{|c|c|c|c|c|c|}
\hline & Overall $(\mathrm{n}=\mathbf{2 2 , 2 5 8})$ & Aprotinin $(n=7329)$ & TXA $(n=1486)$ & $\operatorname{ACA}(n=1667)$ & No drug $(n=11,766)$ \\
\hline Age at operation, mo & $7.6(2.6-43.4)$ & $8.1(2.3-46.0)$ & $6.5(3.5-30.9)$ & $7.9(3.2-49.1)$ & $7.6(2.5-42.8)$ \\
\hline Sex, male & $12,431(55.9 \%)$ & $4121(56.2 \%)$ & $837(56.3 \%)$ & $935(56.1 \%)$ & $6538(55.9 \%)$ \\
\hline Race, white & $15,729(70.7 \%)$ & $5272(71.9 \%)$ & $1064(71.6 \%)$ & $1250(75.0 \%)$ & $8143(69.2 \%)$ \\
\hline Weight, kg & $6.9(4.2-14.5)$ & $7.1(4.1-14.8)$ & $6.4(4.6-12.4)$ & $7.0(4.4-15.1)$ & $6.9(4.2-14.4)$ \\
\hline Weight-for-age z-score & $-1.1(-2.1,-0.2)$ & $-1.1(-2.1,-0.2)$ & $-1.3(-2.3,-0.3)$ & $-1.2(-2.1,-0.2)$ & $-1.1(-2.2,-0.2)$ \\
\hline Noncardiac/genetic abnormality & $6376(28.7 \%)$ & $1982(27.0 \%)$ & $496(33.4 \%)$ & $538(32.3 \%)$ & $3360(28.5 \%)$ \\
\hline Prematurity* & $574(14.0 \%)$ & $215(14.4 \%)$ & $26(12.6 \%)$ & $37(13.6 \%)$ & $296(13.9 \%)$ \\
\hline Any STS preop risk factor & $4955(22.3 \%)$ & $1599(21.8 \%)$ & $357(24.0 \%)$ & $402(24.1 \%)$ & $2597(22.1 \%)$ \\
\hline Previous CT surgery & $7232(32.5 \%)$ & $2909(39.7 \%)$ & $554(37.3 \%)$ & $639(38.3 \%)$ & $3130(26.6 \%)$ \\
\hline \multicolumn{6}{|l|}{ STS-EACTS risk category } \\
\hline 1 & $5465(24.6 \%)$ & $1496(20.4 \%)$ & $333(22.4 \%)$ & $367(22.0 \%)$ & $3269(27.8 \%)$ \\
\hline 2 & $6354(28.6 \%)$ & $2177(29.7 \%)$ & $428(28.8 \%)$ & $499(29.9 \%)$ & $3250(27.6 \%)$ \\
\hline 3 & $4112(18.5 \%)$ & $1364(18.6 \%)$ & $319(21.5 \%)$ & $352(21.1 \%)$ & $2077(17.6 \%)$ \\
\hline 4 & $5063(22.8 \%)$ & $1786(24.4 \%)$ & $350(23.6 \%)$ & $355(21.3 \%)$ & $2572(21.8 \%)$ \\
\hline 5 & $1264(5.7 \%)$ & $506(6.9 \%)$ & $56(3.8 \%)$ & $94(5.6 \%)$ & $608(5.2 \%)$ \\
\hline Periop corticosteroids & $13,890(62.4 \%)$ & $5321(72.6 \%)$ & $1301(87.6 \%)$ & $1243(74.6 \%)$ & $6025(51.2 \%)$ \\
\hline $\begin{array}{l}\text { Annual center volume, median } \\
\text { operations/y }\end{array}$ & $376(328-649)$ & $370(324-501)$ & $649(350-708)$ & $376(342-398)$ & $393(341-649)$ \\
\hline
\end{tabular}

Data are displayed as frequency and percent for dichotomous variables and median and interquartile range for continuous variables. $T X A$, Tranaxemic acid; $A C A$, aminocaproic acid; STS, The Society of Thoracic Surgeons; CT, cadiothoracic; EACTS, European Association for Cardiothoracic Surgery. *Neonates only.

perioperative corticosteroids. Models for the neonatal subgroup were also adjusted for prematurity. Our analytic approach was chosen specifically to account for confounding by center, inasmuch as it was noted from initial inspection of the data that choice of treatment appeared largely center dependent, and historically it has been shown that both hospital care and outcome can vary by center. For dichotomous outcome variables, conditional logistic regression stratified by center was used to account for potential confounding effect of center (this methodology also accounts for potential differences in center volume). Odds ratios and $95 \%$ confidence intervals are reported. For continuous outcome variables, linear regression with center modeled as a main effect was used. Length of stay and duration of ventilation were not normally distributed and were log transformed for analysis. Regression coefficients from the linear regression models were exponentiated, and the ratio of length of stay or duration of ventilation between groups with $95 \%$ confidence intervals (CIs) were reported. In cases in which there was a significant difference between groups, estimates of the difference in actual days at the median value of length of stay or duration of ventilation were also calculated. All analyses were performed in the overall cohort and in 2 selected subgroups: (1) neonates and (2) those with a history of cardiothoracic surgery undergoing redo sternotomy.

Outcomes were first evaluated in those who received aprotinin versus no drug from 2004 to 2007 (the time period when aprotinin was commonly used). Univariable and multivariable analyses were performed as outlined above. Next, the second analysis focused on the years 2007 and 2008 when there was a shift in use of aprotinin to use of ACA or TXA after aprotinin was taken off the market. Outcomes associated with ACA and TXA were compared with aprotinin in univariable and multivariable analysis, again as outlined earlier.

An instrumental variable analysis was then performed as an additional method for comparative analysis of the 3 drugs, in an attempt to further minimize potential confounding center effects. For this analysis, data from 2008 were compared with those from 2007, restricting to centers that switched primarily ( $>75 \%$ use) from aprotinin to ACA, from aprotinin to TXA, and patients at these centers who received any antifibrinolytic medication. All analyses were performed using SAS version 9.2 software (SAS Institute, Inc, Cary, NC). Patients with missing data for a study end point were excluded from analysis involving that end point. The $P$ values presented are without adjustment for multiple comparisons.

\section{RESULTS}

\section{Study Population Characteristics}

A total of 22,258 patients ( 25 centers) were included. Patient characteristics overall and for those who received each antifibrinolytic medication are displayed in Table 1 . Change in medication use patterns is displayed in Figure 1. During 2004 to $2007,49 \%$ of patients overall received an antifibrinolytic medication (42\% aprotinin, $7 \%$ ACA or TXA). In 2008, after aprotinin was taken off the market, a similar proportion of patients overall received an antifibrinolytic medication ( $43 \%$ ), but only $5 \%$ received aprotinin whereas $20 \%$ received ACA and $18 \%$ received TXA. Overall, during the study period, 23 centers used aprotinin, 20 centers used ACA, and 11 centers used TXA. Of note, mortality in 2004 to 2007 versus 2008 was similar $(3.6 \%$ vs $3.4 \%)$.

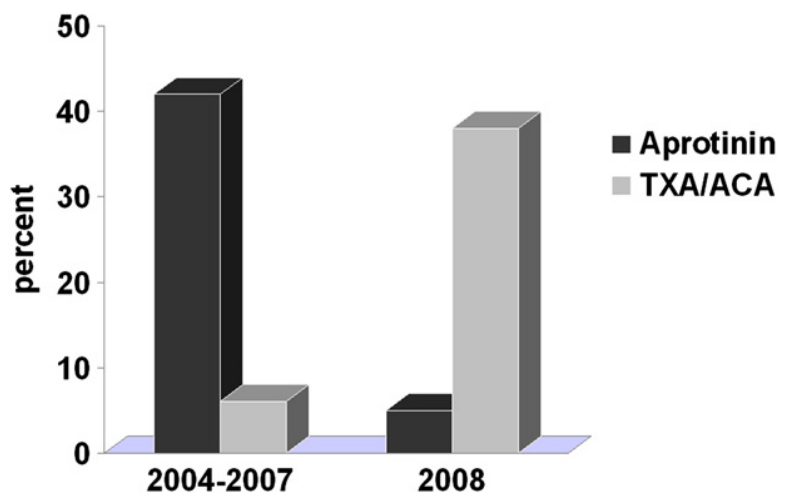

FIGURE 1. Antifibrinolytic use in children undergoing heart surgery. $T X A$, Tranaxemic acid; $A C A$, aminocaproic acid. 
TABLE 2. Unadjusted and adjusted postoperative outcomes associated with aprotinin versus no drug

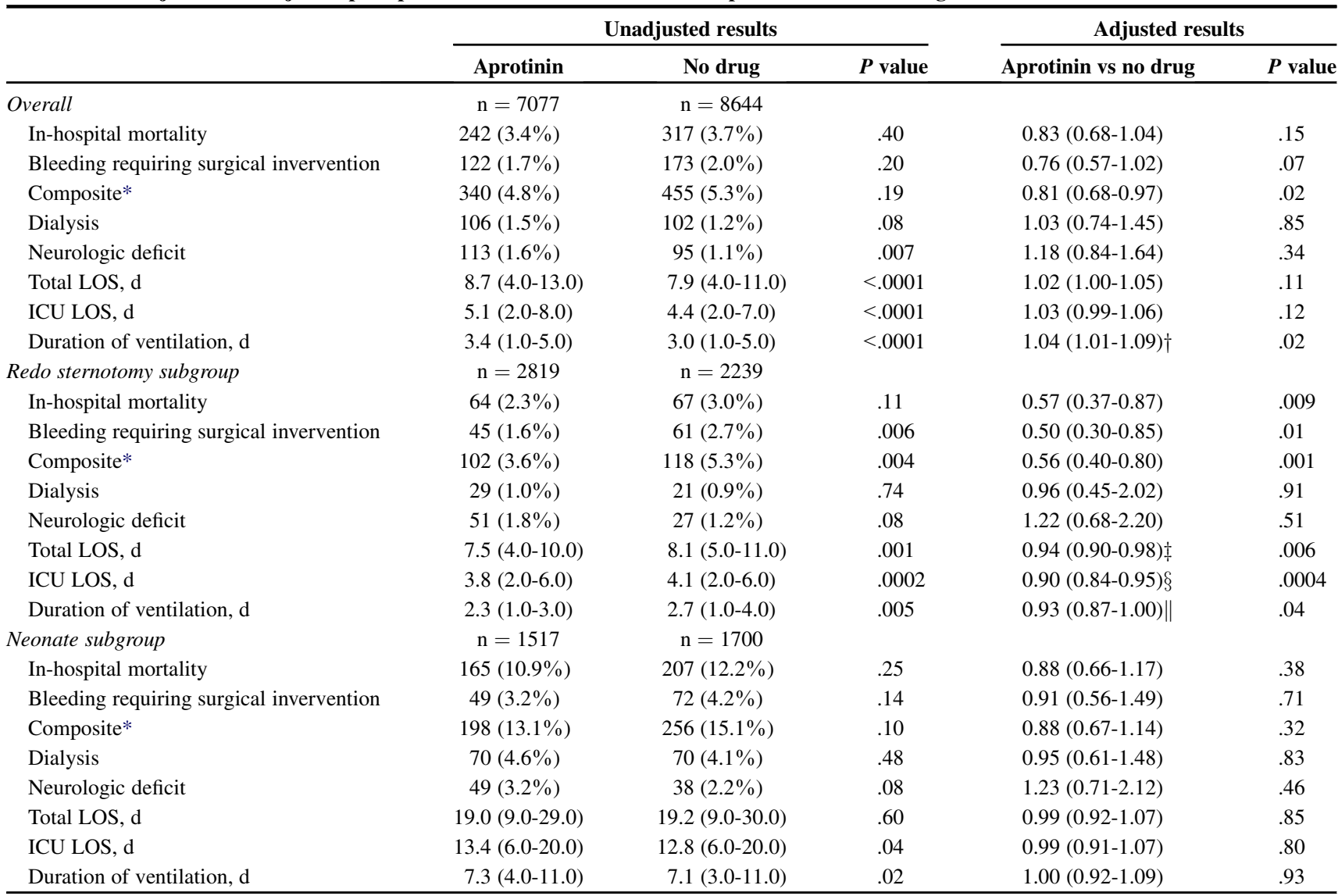

$L O S$, Length of stay; $I C U$, intensive care unit. *Composite end point $=$ in-hospital mortality or bleeding requiring reoperation. Unadjusted results are displayed as frequency (percent) for dichotomous variables and 10\% trimmed means (interquartile range) for continuous variables. Adjusted results (from conditional logistic regression for dichotomous variables and linear regression with center modeled as a main effect for continuous variables) are displayed as adjusted odds ratio ( $95 \%$ confidence intervals) for dichotomous variables and adjusted ratio of LOS or duration of ventilation (days) between groups for continuous variables. In those groups in which aprotinin was associated with a significant difference in LOS or duration of ventilation, adjusted estimates of the difference in actual days (aprotinin vs no drug) at the median value of LOS or duration of ventilation were also calculated: †Overall group, duration of ventilation ( +0.1 days); reoperation subgroup; łtotal LOS ( -0.5 days); §ICU LOS $(-0.3$ days); $\|$ duration of ventilation $(-0.1$ days $)$.

Subsequent analyses were performed both in the overall cohort of patients and in 2 subgroups: neonates $(\mathrm{n}=4426)$ and those undergoing redo sternotomy $(\mathrm{n}=7232)$. The most common procedures performed in the neonate subgroup were Norwood operation (23\%), arterial switch operation $(23 \%)$, coarctation/interrupted aortic arch repair (13\%), repair of total anomalous pulmonary venous connection $(10 \%)$, and truncus arteriosus repair $(4 \%)$. The most common procedures performed in the reoperation subgroup were Fontan operation (17\%), bidirectional cavopulmonary anastomosis $(14 \%)$, conduit operation $(10 \%)$, pulmonary valve replacement $(4 \%)$, and subvalvular aortic stenosis repair $(4 \%)$.

\section{Aprotinin Versus No Drug}

We first evaluated outcomes associated with aprotinin versus no drug in 2004 to 2007, the time period when aprotinin was most commonly used. Unadjusted and adjusted outcomes are displayed in Table 2. In multivariable analysis, aprotinin was associated with a significant reduction in the composite end point of in-hospital mortality/ bleeding requiring surgical intervention in the overall cohort. There was a statistically significant difference between groups in duration of ventilation (0.1 days), likely not clinically meaningful (Table 2). There were no other differences in outcome detected in the overall cohort including no difference in renal failure requiring dialysis.

In the redo sternotomy subgroup, aprotinin was associated with a significant reduction in mortality and bleeding requiring surgical intervention, as well as reduced length of total and intensive care unit length of stay. No differences were detected in dialysis or neurologic complications (Table 2). In the neonatal subgroup, aprotinin was not associated with a significant difference in any outcome evaluated (Table 2).

\section{Aprotinin Versus TXA and ACA}

Efficacy and safety outcomes were then compared in patients who received aprotinin, TXA, or ACA in 2007 and 
TABLE 3. Unadjusted and adjusted postoperative outcomes associated with TXA, ACA, and aprotinin

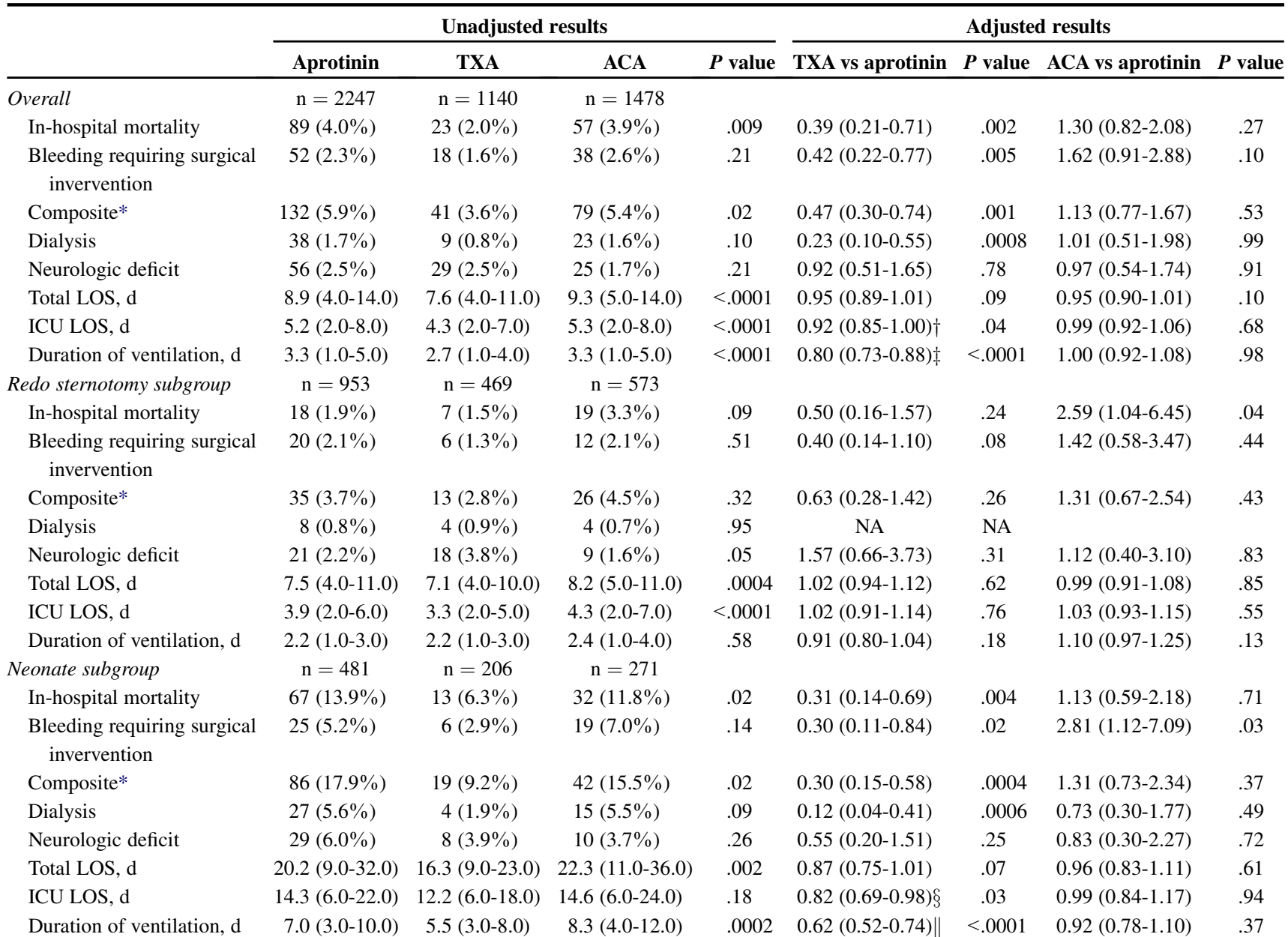

TXA, Tranaxemic acid; $A C A$, aminocaproic acid; $N A$, not enough events to model. ${ }^{*}$ Composite end point $=$ in-hospital mortality or bleeding requiring reoperation. Unadjusted results are displayed as frequency (percent) for dichotomous variables, and $10 \%$ trimmed means (interquartile range) for continuous variables. Adjusted results (from conditional logistic regression for dichotomous variables and linear regression with center modeled as a main effect for continuous variables) are displayed as adjusted odds ratio (95\% confidence intervals) for dichotomous variables and adjusted ratio of LOS or duration of ventilation (days) between groups for continuous variables. In those groups in which aprotinin was associated with a significant difference in LOS or duration of ventilation, adjusted estimates of the difference in actual days at the median value of LOS or duration of ventilation were also calculated: Overall group: †ICU LOS, TXA vs aprotinin ( -0.3 days); $\ddagger$ duration of ventilation, TXA vs aprotinin $(-0.4$ days). Neonate subgroup: $\S$ ICU LOS, TXA vs aprotinin ( -1.8 days); ||duration of ventilation, TXA vs aprotinin ( -2.3 days).

2008 (the time period when use shifted from aprotinin to TXA or ACA) in unadjusted and adjusted analysis (Table 3). In multivariable analysis, there was generally no difference detected in efficacy or safety outcomes comparing aprotinin with ACA overall or in the 2 subgroups, with the exception of greater in-hospital mortality associated with ACA in the reoperation subgroup and greater bleeding requiring surgical invervention in the neonatal subgroup (Table 3 ). In contrast, TXA was generally associated with improved outcomes in comparison with aprotinin. This included reduced in-hospital mortality, bleeding requiring surgical invervention, intensive care unit length of stay, and dialysis associated with TXA versus aprotinin in the overall cohort (Table 3 ). In the neonatal subgroup, similar differences were seen when comparing TXA with aprotinin. In the redo sternotomy subgroup, no significant differences between TXA and aprotinin were detected (Table 3).

\section{Instrumental Variable Analysis}

An instrumental variable analysis was performed as a second method for comparative analysis of the 3 drugs in an attempt to further minimize potential confounding center effects. Data from 2008 were compared with 2007, restricted to centers that switched primarily ( $>75 \%$ use) to ACA from aprotinin ( $\mathrm{n}=7 ; 1553$ patients) or switched primarily to TXA from aprotinin ( $\mathrm{n}=3 ; 653$ patients) and patients who received any antifibrinolytic medication at these centers. Owing to the smaller number of patients, only the composite end point could be modeled in the overall cohort in multivariable analysis. Among centers that switched primarily to TXA from aprotinin, there was not a significant 
difference detected in in-hospital mortality or bleeding requiring surgical intervention in 2008 (primarily TXA) versus 2007 (primarily aprotinin): adjusted odds ratio (OR), 0.53 (95\% CI, $0.27-1.05 ; P=.07)$. However, the point estimate was in the same direction as the primary analysis noted earlier, favoring TXA. Among centers that switched primarily to ACA from aprotinin, there was no difference detected in in-hospital mortality or bleeding requiring surgical intervention in 2008 (primarily ACA) versus 2007 (primarily aprotinin): adjusted OR, 0.85 (95\% CI, 0.52$1.41 ; P=.54)$. There were too few dialysis events to model in multivariable analysis; however, in unadjusted analysis in centers that switched primarily to TXA from aprotinin, there was a reduction in renal failure requiring dialysis in 2008 (primarily TXA) versus 2007 (primary aprotinin): $0.7 \%$ versus $3.1 \% ; P=.03$. In centers that switched primarily to ACA from aprotinin, there was not a significant difference detected in the proportion with renal failure requiring dialysis in 2008 (primarily ACA) versus 2007 (primarily aprotinin): $1.2 \%$ versus $2.7 \% ; P=.07$. The overall number of patients requiring dialysis in both cases was small (9 and 32, respectively).

\section{DISCUSSION}

This multicenter contemporary analysis of antifibrinolytic medications in children undergoing heart surgery suggests aprotinin is associated with reduced bleeding requiring surgical intervention and mortality with no increase in dialysis. Comparative analyses suggest similar efficacy of ACA and improved outcomes associated with TXA.

Several previous studies of aprotinin in the pediatric population have suggested aprotinin is effective in reducing bleeding after heart surgery, and a meta-analysis of 12 randomized controlled trials $(n=626)$ found that aprotinin reduced the proportion of children who received transfusions during cardiac surgery by $33 \% .{ }^{14}$ Our analysis of a contemporary cohort of more than 20,000 children suggests that aprotinin is associated with reduced bleeding requiring reoperation and mortality. These effects were most prominent in the subgroup of patients undergoing redo sternotomy. Previous analyses have suggested differential efficacy of aprotinin, particularly in those at highest risk for bleeding. ${ }^{7}$ Costello and colleagues ${ }^{7}$ found that only those undergoing reoperation had a significant decrease in transfusions associated with aprotinin in an observational study of 112 children undergoing heart surgery. Previous studies have also reported a reduction in inflammatory markers in patients who receive aprotinin, as well as a reduction in postoperative myocardial dysfunction and inotropic support. ${ }^{15-17}$ These properties, along with the impact of aprotinin on bleeding, may have contributed to the decreased length of stay and duration of mechanical ventilation we observed in the redo sternotomy subgroup.
However, many of the previous investigations have been limited by important methodologic concerns including small sample size, heterogeneous patient population, lack of standardized transfusion protocols, and varying doses of aprotinin used, and not all studies have demonstrated a beneficial effect of aprotinin. ${ }^{14}$ In addition, in a previous analysis of safety outcomes conducted by our group using an administrative data source, we did not find a significant impact of aprotinin on mortality overall or in those undergoing reoperation. ${ }^{4}$ Analyses involving administrative data must rely on ICD-9 codes from the hospital bill to identify children undergoing heart surgery. In contrast, in the present study we were able to use data from a clinical registry to identify the study population. Using our linked data set, which contains both the clinical registry data in the STSCHS Database and the ICD-9 codes from the administrative database (PHIS) for each patient, we found that $10 \%$ of our overall study cohort would not have been identified if we had relied on ICD-9 codes alone, including $18 \%$ of patients in the redo sternotomy cohort and $13 \%$ of patients in higher STS-EACTS risk categories (categories 3-5) versus $6 \%$ of patients in lower STS-EACTS risk categories (categories $1-2$ ). It is possible that these differences may in part explain differences in study results.

Regarding the safety of aprotinin, we did not find any difference in renal failure requiring dialysis in comparison with no drug. Our data support results of previous singlecenter observational analyses. Guzzetta and colleagues ${ }^{18}$ evaluated 200 neonates undergoing surgery for congenital heart disease and found that aprotinin was not significantly associated with postoperative creatinine levels or dialysis. Backer and associates ${ }^{19}$ evaluated 1251 children and adolescents undergoing surgery for congenital heart defects and found that aprotinin was not associated with postoperative renal failure or dialysis compared with historical controls. Evaluation of safety was limited in the small randomized trials of aprotinin in children, and in the recent meta-analysis combining results from 12 trials, mortality and renal failure could not be assessed. ${ }^{14}$

In comparative analyses, our observational data suggested that, in general, ACA was associated with similar or worse outcome than was aprotinin. There are few previous studies comparing outcomes in patients receiving different antifibrinolytic medications, and it is difficult to draw conclusions owing to differences in study design, dosing, outcomes, and small sample sizes. Chauhan and colleagues $^{5}$ compared aprotinin with ACA in 300 children undergoing heart surgery and found no difference in blood loss, transfusion, and need for re-exploration.

In contrast, we found that TXA appeared to be associated with better outcomes than aprotinin. These results differ from a previous analysis of 100 children undergoing heart surgery that found no difference in blood loss or transfusion in those randomized to aprotinin versusTXA. ${ }^{6}$ This study 
included fewer patients than did our analysis and evaluated different outcomes. The reasons for potentially greater efficacy of TXA are unclear. There are limited pediatric pharmacokinetic data available, and in our study we were unable to evaluate medication doses. It has been previously reported that TXA may be a more potent inhibitor of fibrinolysis than ACA. ${ }^{20,21}$ In addition, TXA may have antiinflammatory properties. ${ }^{22}$

In regard to safety, we did not detect any difference comparing ACA with aprotinin. TXA appeared to be associated with a lower rate of dialysis than did aprotinin. However, the overall number of events was small and it may be premature to draw any conclusions from these data. There were also few neurologic events in our study. Nonetheless, we did not find any difference comparing ACA, TXA, and aprotinin. Previous case reports have suggested that TXA may be associated with an increase in postoperative seizures in adult patients undergoing cardiac surgery. ${ }^{13}$

\section{Limitations}

This study is subject to the limitations of all observational analyses. Our analytic strategy attempted to account for known patient confounders; however, it is likely that there are other variables not captured in these databases that may affect both the receipt of antifibrinolytic medication and outcome. These may include differences in bypass strategies. We also attempted to account for center effects through our analytic approach using conditional logistic regression and instrumental variable analysis. However, these strategies may not account for all differences between centers, and our results may not be generalizable to all centers performing surgery for congenital heart defects.

Our study is also subject to the limitations of the databases. Although our large sample size, multicenter data, and combined data from both a clinical registry and administrative database allowed evaluation of end points such as mortality and other safety outcomes not able to be assessed in previous single-center studies, we were not able to evaluate other outcomes such as volume of blood loss, transfusions, and more mild degrees of renal impairment, inasmuch as these data are not collected in the databases used. In addition, the data sources used for this study also do not contain information on dosing or indication for administration. Thus, we were not able to compare specific doses of antifibrinolytic medications. Rather, these data represent outcomes associated with these medications in routine clinical practice across multiple centers.

Finally, although this analysis represents the largest evaluation of these medications in the pediatric population, the small sample size in certain subgroups, as well as the small number of events, limited our analysis and power to detect differences between groups in some cases. For example, we were unable to perform adjusted analyses of dialysis in the instrumental variable analysis; thus these data are not adjusted for patient risk and must be interpreted with caution. In addition, our analysis involved multiple comparisons. We chose not to adjust the $P$ values or to specify a $P$ value cutoff for "significance" inasmuch as this methodology can be somewhat subjective; however, we cannot rule out the possibility that some of our findings may be due to chance alone.

\section{CONCLUSIONS}

This large observational analysis of children undergoing heart surgery suggests that aprotinin is associated with reduced bleeding requiring surgical intervention and mortality with no increase in dialysis in comparison with no drug. Comparative analyses suggest similar efficacy of ACA and improved outcomes associated with TXA. These findings should be evaluated in an adequately powered clinical trial.

\section{References}

1. Fergusson DA, Hebert PC, Mazer CD, Fremes S, MacAdams C, Murkin JM, et al. A comparison of aprotinin and lysine analgues in high-risk cardiac surgery. N Engl J Med. 2008;358:2319-31.

2. Mangano D, Tudor JC, Dietzel C. The risk associated with aprotinin in cardiac surgery. N Engl J Med. 2006;354:353-65.

3. Petaja J, Lundstrom U, Leijala M, Peltola K, Siimes MA. Bleeding and use of blood products after heart operations in infants. $J$ Thorac and Cardiovas Surg. 1995;109:524-9.

4. Pasquali SK, Hall M, Li JS, Peterson ED, Jaggers J, Lodge AJ, et al. Safety of aprotinin in congenital heart operations: results from a large multicenter database. Ann Thorac Surg. 2010;90:14-21.

5. Chauhan S, Kumar BA, Rao BH, Rao MS, Dubey B, Saxena N, et al. Efficacy of aprotinin, epsilon aminocaproic acid, or combination in cyanotic heart disease. Ann Thorac Surg. 2000;70:1308-12.

6. Bulutcu FS, Ozbek U, Polat B, Yalcin Y, Karaci AR, Bayindir O. Which may be effective to reduce blood loss after cardiac operations in cyanotic children: tranexamic acid, aprotinin or a combination? Paediatr Anaesth. 2005;15:41-6.

7. Costello JM, Backer CL, de Hoyos A, Binns HJ, Mavroudis C. Aprotinin reduces operative closure time and blood product use after pediatric bypass. Ann Thorac Surg. 2003;75:1261-6.

8. Pasquali SK, Jacobs JP, Shook GJ, O’Brien SM, Hall M, Jacobs ML, et al. Linking clinical registry data with administrative data using indirect identifiers: implementation and validation in the congenital heart surgery population. Am Heart J. 2010;160:1099-104

9. Franklin RC, Jacobs JP, Krogmann ON, Beland MJ, Aiello VD, Colan SD, et al. Nomenclature for congenital and paediatric cardiac disease: historical perspectives and The International Pediatric and Congenital Cardiac Code. Cardiol Young. 2008;18(Suppl 2):70-80.

10. Clarke DR, Breen LS, Jacobs ML, Franklin RC, Tobota Z, Maruszewski B, et al. Verification of data in congenital cardiac surgery. Cardiol Young. 2008; 18(Suppl 2):177-87

11. O'Brien SM, Clarke DR, Jacobs JP, Jacobs ML, Lacour-Gayet FG, Pizarro C, et al. An empirically based tool for analyzing mortality associated with congenital heart surgery. J Thorac Cardiovasc Surg. 2009;138:1139-53.

12. Society of Thoracic Surgeons. STS congenital database full specifications. Available at: http://www.sts.org/documents/pdf/Congenital_DataSpecs_250. pdf. Accessed November 15, 2010.

13. Murkin JM, Falter F, Granton J, Young B, Burt C, Chu M. High-dose tranexamic acid is associated with nonischemic clinical seizures in cardiac surgical patients. Anesth Analg. 2010;110:350-3.

14. Arnold DM, Fergusson DA, Chan AK, Cook RJ, Faser GA, Lim W, et al. Avoiding transfusions in children undergoing cardiac surgery: a meta-analysis of randomized trials of aprotinin. Anesth Analg. 2006;102:731-7. 
15. Tassani P, Augustin N, Barankay A, Braun SL, Zaccaria F, Richter JA. High dose aprotinin modulates the balance between proinflammatory and antiinflammatory response during coronary artery bypass graft surgery. J Cardiovasc Thorac Surg. 2000;14:682-6.

16. Wippermann CF, Schmid FX, Eberle B, Huth RG, Kampmann C, Schranz D, et al. Reduced inotropic support after aprotinin therapy during pediatric cardiac operations. Ann Thorac Surg. 1999;67:173-6.

17. Tweddell J, Berger S, Frommelt PC, Pelech AN, Lewis DA, Fedderly RT, et al. Aprotinin improves outcome of single ventricle palliation. Ann Thorac Surg. 1996;62:1329-36.

18. Guzzetta NA, Evans FM, Rosenberg ES, Fazlollah TM, Baker MJ, Wilson EC, et al. The impact of aprotinin on postoperative renal dysfunction in neonates undergoing cardiopulmonary bypass. Anesth Analg. 2009;108:448-55.
19. Backer CL, Kelle AM, Stewart RD, Suresh SC, Ali FN, Cohn RA, et al. Aprotinin is safe in pediatric patients undergoing cardiac surgery. $J$ Thorac Cardiovasc Surg. 2007; 134:1421-6.

20. Fiechtner BK, Nuttall GA, Johnson ME, Dong Y, Sujirattanawimol N, Oliver WC, et al. Plasma tranexamic acid concentrations during cardiopulmonary bypass. Anesth Analg. 2001;92:1131-6.

21. McNicol G, Fletcher A, Alkjaersig N, Sherry S. The absorption, distribution, and excretion of epsilon-aminocaproic acid following oral or intravenous administration to man. J Lab Clin Med. 1962;59:15-24.

22. Jimenez JJ, Iribarren JL, Lorente L, Rodriguez JM, Hernandez D, Nassar I, et al Tranexamic acid attenuates inflammatory response in cardiopulmonary bypass surgery through blockade of fibrinolysis: a case control study followed by a randomized double-blind controlled trial. Crit Care. 2007;11:R117. 